\title{
Association between neurocognition and social cognition in a sample of people with schizophrenia spectrum disorders
}

A. Munoz-Sanjose ${ }^{1,2}$, A. De Diego ${ }^{1}$, R. Mediavilla ${ }^{2,3}$, A. Orosa ${ }^{1}$, M.P. Vidal-Villegas ${ }^{2}$, N. Fernandez ${ }^{1}$, A. Garcia ${ }^{1}$, G. Kollias ${ }^{1}$, J.M. Pastor ${ }^{1}$, C. Bayon ${ }^{1,2}$, S. Cebolla ${ }^{1}$, G. Lahera $^{4,5}$, A. Palao ${ }^{1,2}$, B. Rodriguez-Vega ${ }^{1,2}$, E. Roman ${ }^{1}$, P. Sanchez ${ }^{1}$ A. Pinto ${ }^{2}$, E. Jiménez ${ }^{1}$, N. Millán ${ }^{1}$, M.F. Bravo-Ortiz ${ }^{1,2}$

1 La Paz University Hospital, Psychiatry- Clinical Psychology and Mental Health, Madrid, Spain.

2 Hospital La Paz Institute for Health Research (IdiPAZ), Psychiatry and Mental Health Group, Madrid, Spain.

3 National University of Distance Education UNED, Psychology, Madrid, Spain.

4 University of Alcala, Faculty of Medicine. Psychiatry Department, Alcala de Henares, Spain

5 CIBERSAM, Mental Health, Madrid, Spain.

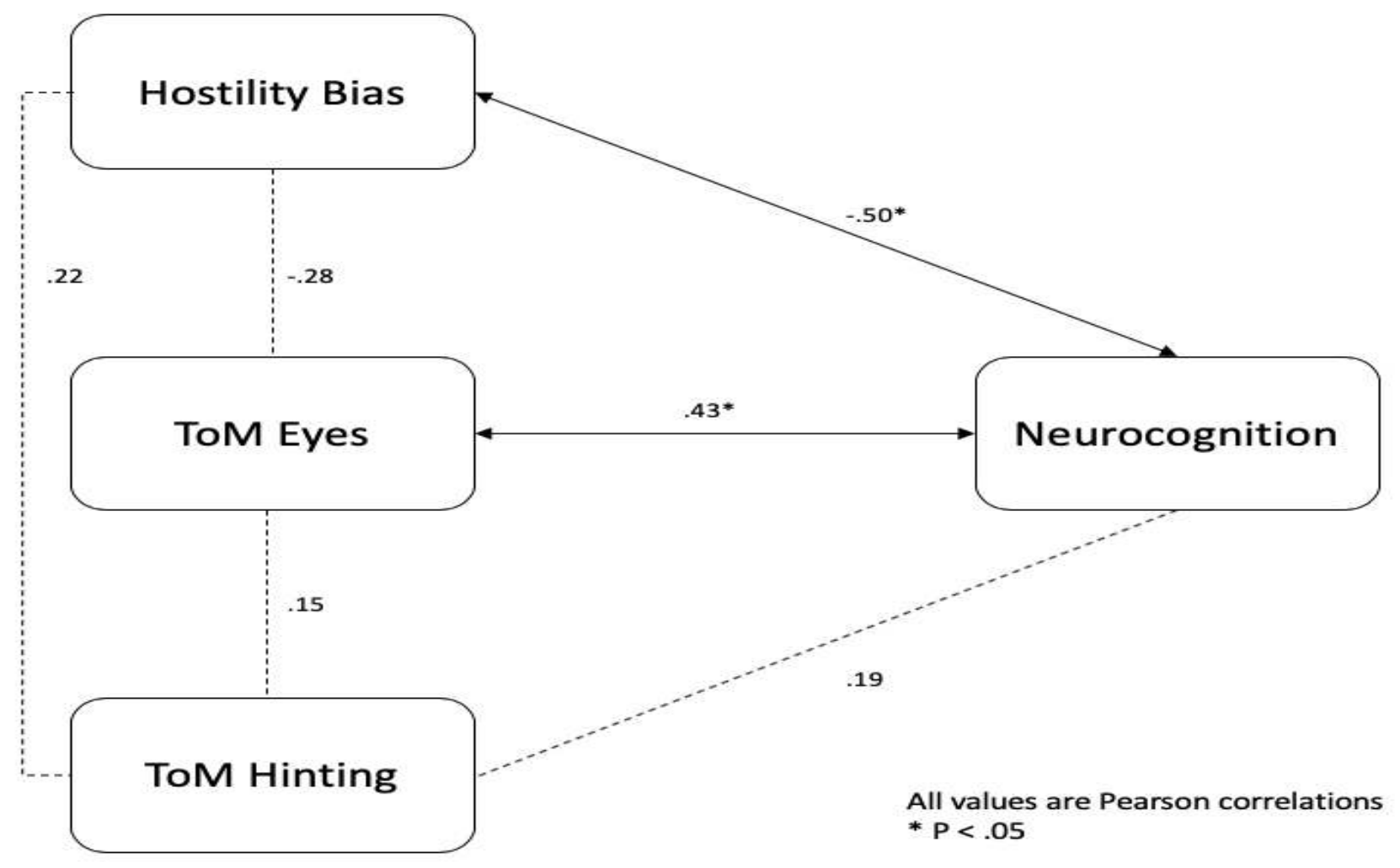

Background

Neurocognition and social cognition are domains often affected in schizophrenia spectrum disorders (SSDs) (Green et al., 2015, Bora et al., 2017). Despite the important number of psychological interventions addressing them (Grant et al., 2017, Cella et al., 2017), it is still not clear if neurocognition and social cognition are associated, or what their functional implications are (Mehta et al., 2013; Schmidt et al., 2011).

\section{Objective}

To check if neurocognition and social cognition are linked in a sample of people with SSD to tailor clinical interventions

\section{Material and methods}

A sample of $\mathbf{2 5}$ participants with schizophrenia and schizoaffective disorders was included. They were assessed within one week in September 2018. Social cognition measures were taken according to the recommendations of the Social Cognition Psychometric Evaluation (SCOPE) panel. Theory of mind was measured with the short version of the Hinting Task and with the Reading the Mind in the Eyes Test (RMET). Attributional Style was measured with the Attribution of Intentions and Hostility Questionnaire (AIHQ). Assessment of neurocognition was made with the Screen for Cognitive Impairment in Psychiatry (SCIP-S).
Scan for supplementary material:

Results are shown in Figure 1. Higher levels of neurocognition were associated with higher emotional theory of mind and with lower hostile attributions. Neurocognition and cognitive theory of mind were not correlated. These results suggest that neurocognition and social cognition are linked in SSDs.

Further, each domain of social cognition seems to be partly independent from another. Clinical interventions should combine both cognitive remediation and social cognition training.

Bora, E., Akdede, B. B., \& Alptekin, K. (2017). Neurocognitive impairment in deficit and non-deficit schizophrenia: A meta-analysis. Psychological Medicine, https://doi.org/10.1017/S0033291717000952

Cella, M., Preti, A., Edwards, C., Dow, T., \& Wykes, T. (2017). Cognitive remediation for negative symptoms of schizophrenia : A network meta-analysis. Clinical Psychology Review, 52, 43-51. https://doi.org/10.1016/j.cpr.2016.11.009

Grant, N., Lawrence, M., Preti, A., Wykes, T., \& Cella, M. (2017). Social cognition interventions for people with schizophrenia: a systematic review focussing on methodological quality and intervention modality. Clinical Psychology Review, 56(August), 55-64. https://doi.org/10.1016/j.cpr.2017.06.001 Green, M. F., Horan, W. P., \& Lee, J. (2015). Social cognition in schizophrenia. Nature Reviews Neuroscience, 16(10), 620-631. https://doi.org/10.1038/nrn4005

Mehta, U. M., Thirthalli, J., Naveen Kumar, C., Mahadevaiah, M., Rao, K., Subbakrishna, D. K.,... Keshavan, M. S. (2011). Validation of social cognition rating tools in indian setting (SOCRATIS): A new test-battery to assess social cognition. Asian Journal of Psychiatry, 4(3), 203-209. https://doi.org/10.1016/j.ajp.2011.05.014 